\title{
Electrophoresis- and FRET-Based Measures of Serpin Polymerisation
}

\author{
Sarah V. Faull ${ }^{1}$, Anwen E. Brown², Imran $\mathrm{Haq}^{2}$ and James A. \\ Irving ${ }^{2}$
}

1Division of Structural Biology, The Institute of Cancer Research, London SW3 3RP, UK. 2UCL Respiratory, University College London, London WC1E 6JF, UK.

\section{Summary}

Many serpinopathies, including alpha-1-antitrypsin (A1AT) deficiency, are associated with formation of unbranched polymer chains of mutant serpins. In vivo, this is the result of mutations that cause kinetic or thermodynamic destabilisation of the molecule. However, polymerisation can also be induced in vitro from mutant or wild-type serpins under destabilising conditions. The characteristics of the resulting polymers are dependent upon induction conditions. Due to their relationship to disease, serpin polymers, mainly those formed from A1AT, have been widely studied. Here we describe FRET and gel-based approaches for their characterisation.

Key words: Polymerisation, Protein Aggregation, Kinetics, Monoclonal antibody, Immunoblots

\section{Introduction}

The serpinopathies are a group of hereditary diseases associated with pathological gain-of-function or loss-of-function phenotypes. The underlying bases of many of these conditions are the misfolding and accumulation of mutant serpins, including alpha-1-antitrypsin (A1AT) (Gooptu and Lomas, 2009). In vivo, mutant A1AT forms linear, flexible unbranched chains of molecules with a 'beads-on-a-string' morphology when visualised by EM (Lomas et al., 1992; Lomas et al., 1993). These are referred to as polymers. Their accumulation results in the deposition of material at the site of synthesis, observable in ex vivo samples as diastase resistant and Periodic Acid Schiff positive inclusions within hepatocytes (Berg and Eriksson, 1972). The consequence of this deposition is physical distension of the endoplasmic reticulum, where the protein is synthesised as the first step in the secretory pathway, with consequences for intraluminal mobility (Ordóñez et al., 2013). However, polymer formation does not trigger the unfolded protein response associated with an accumulation of misfolded protein (Hidvegi et al., 2005; Kröger et al., 2009; Ordóñez et al., 2013).

The structural configurations adopted by serpins can be classified into two distinct states: (1) the kinetically stable but thermodynamically unstable native conformation, with a 5 -stranded central $\beta$-sheet $A$ and exposed reactive centre loop; and (2) the thermodynamically hyperstable 6-stranded $\beta$-sheet A 'reactive centre loop incorporated' 
conformation (Bruch et al., 1988; Carrell and Owen, 1985). It is the latter configuration that subunits within the polymer are proposed to adopt (Lomas et al., 1992; Yamasaki et al., 2008; Yamasaki et al., 2011). Correspondingly, polymers exhibit extreme stability (Mast et al., 1992).

Core to the understanding of the mechanism of polymerisation is the development of methods capable of inducing it under controlled conditions in vitro. Their utility arises in part from a relative scarcity of explant liver material, and they also provide a means to interrogate or manipulate polymer formation as a kinetic process. These methods have typically involved the use of conditions that destabilise the native state: (1) denaturant (Lomas et al., 1992); (2) heat (Mast et al., 1992); or (3) acidic pH (Devlin et al., 2002). However, different conditions result in polymers that are distinct immunologically (Ekeowa et al., 2010; Miranda et al., 2010) and in structural character (Devlin et al., 2002; Yamasaki et al., 2011), findings which suggest different polymer forms are accessible using in vitro methods. This has likely been a contributing factor in the emergence of several apparently inconsistent models of the pathological A1AT polymer (Lomas et al., 1992; Lomas et al., 1993; Yamasaki et al., 2008; Yamasaki et al., 2011). Consistent with the observation that peptides based on this region were found to antagonise the polymerisation process (Lomas et al., 1992), and polymers show comparable stability to the loop-incorporated form (Powell and Pain, 1992) these models countenance a reactive centre loop embedded in a 6stranded $\beta$-sheet $A$, though differ in their description of the associated intermolecular linkage.

Biochemically, the process of polymerisation can be described by the following scheme (Dafforn et al., 1999):

$$
\begin{aligned}
M(\text { monomer }) & \rightarrow M^{*} \text { (activated monomer) } \\
M^{*}+M^{*} & \rightarrow P \text { (polymer) }
\end{aligned}
$$

in which the 'activated monomer' represents one or more monomeric, oligomerisation-competent (Irving et al., 2015) intermediates on the pathway (Haq et al., 2013).

With the choice of destabilising conditions influencing the nature of the resultant polymers, a key question is the degree to which observations made in vitro can be extended to the pathological context. This is exemplified by the 2C1 monoclonal antibody, which recognises an epitope held in common between pathological material and heatinduced, but not denaturant-induced, in vitro polymers (Ekeowa et al., 2010). Furthermore, there is debate as to whether polymers arise in vivo from a near-native state or a folding intermediate. The latter possibility appears consistent with the observation that the denaturant-mediated unfolding pathway traverses an intermediate ensemble with a tendency to polymerise (Lomas et al., 1992; Powell and Pain, 1992). However, this intermediate shows a measurably lower degree of packing with respect to that imparted during heating (Tsutsui et al., 2012; Tsutsui et al., 2008). This is important, because different studies have made use of these two techniques to draw conclusions about polymerisation processes that may in fact be distinct.

An understanding of the process of polymerisation is of direct relevance to the development of therapeutics (Mallya et al., 2007) and studies 
aimed at characterising or manipulating the cellular response to polymer burden (Ordóñez et al., 2015; Ordóñez et al., 2013). In this chapter, we provide methods for assessing the progress of heat-induced A1AT polymerisation.

\section{Materials}

High purity water denotes $>10 \mathrm{M} \Omega$ resistivity, ultrapure denotes $>18 \mathrm{M} \Omega$ resistivity at $25^{\circ} \mathrm{C}$.

\section{Preparation of fluorescent A1AT}

1. A spectrophotometer capable of measuring absorbance in the UV and visible range.

2. A UV transilluminator/camera assembly, or a fluorescence gel scanner.

3. Disposable centrifugal concentrator units with a cut-off of $10 \mathrm{kDa}$ or $30 \mathrm{kDa}$ (the latter will allow faster concentration).

4. Dialysis membrane with a cut-off between $10 \mathrm{kDa}$ and $30 \mathrm{kDa}$.

5. Syringe or centrifugal filters (with $0.45 \mu \mathrm{m}$ cut-off).

6. Phosphate-buffered saline with sodium azide (PBSZ): $8 \mathrm{~g} \mathrm{NaCl}, 0.2 \mathrm{~g}$ $\mathrm{KCl}, 1.44 \mathrm{~g} \mathrm{Na}_{2} \mathrm{HPO}_{4}, 0.24 \mathrm{~g} \mathrm{KH}_{2} \mathrm{PO}_{4}, 0.2 \mathrm{~g}$ sodium azide, made to a volume of $0.8 \mathrm{~L}$ in ultrapure water, the $\mathrm{pH}$ adjusted as necessary to 7.4 with $\mathrm{HCl}$, volume adjusted to $1 \mathrm{~L}$ with ultrapure water, and filtered through a $0.45 \mu \mathrm{m}$ filter.

7. A $1 \mathrm{M}$ solution of dithiothreitol in ultrapure water, divided into aliquots and frozen.

8. $1 \mathrm{M}$ stock solution of L-cysteine hydrochloride, freshly made with ultrapure water.

9. Two thiol-reactive fluorescent labels, one which will be directly excited (the 'donor') and one indirectly excited (the 'acceptor'). For thiol selectivity and stability, maleimide-based conjugates are recommended (see Note 1). These are diluted to a concentration of $50 \mathrm{mM}$ in DMSO and stored in small aliquots (enough for one estimated use) at -80C; this should be done quickly as conjugates are inactivated by oxidation. In our experience, reactivity is maintained for at least 6 months when prepared in this way.

10. Human plasma A1AT (see Note 2), or human recombinant A1AT with either the wild-type cysteine at position 232 or another solventaccessible cysteine introduced by mutagenesis. Protein should have been purified to homogeneity (see Note 3 ).

\section{Sample preparation using anion exchange chromatography}

1. A chromatography system (pump, fraction collector, disposable tubes) (see Note 4).

2. A compatible $1 \mathrm{ml}$ HiTrap Q column (GE Healthcare) or equivalent strong anion exchange resin.

3. A UV transilluminator/camera assembly, or a fluorescence gel scanner.

4. Anion exchange buffer A: $20 \mathrm{mM}$ Tris $\mathrm{pH} 8.0,0.2 \mathrm{~g}$ sodium azide, made in $1 \mathrm{~L}$ ultrapure water, and filtered through a $0.45 \mu \mathrm{m}$ filter (see Note 5). 
5. Anion exchange buffer B: $20 \mathrm{mM}$ Tris $\mathrm{pH} 8.0,1 \mathrm{M} \mathrm{NaCl}, 0.2 \mathrm{~g}$ sodium azide, made in $1 \mathrm{~L}$ ultrapure water, and filtered through a $0.45 \mu \mathrm{m}$ filter (see Note 5).

\section{Heat-induced polymerisation monitored by FRET}

1. Real-time thermal cycler (see Note 6).

2. Centrifuge with plate holders.

3. Real-time compatible PCR plate and plate sealant.

4. Suitable sample buffer, at the desired $\mathrm{pH}$ and ionic strength, such as PBS.

5. Two derivatives of plasma-derived or recombinant A1AT, one labelled using a 'donor' fluorophore and one labelled using an 'acceptor' fluorophore, at a stock concentration of $1 \mathrm{mg} \mathrm{ml}^{-1}$ or greater in PBSZ.

\section{End-point experiments of polymerisation induced at multiple temperatures}

1. A thermal cycler (fluorescence detection is not necessary) capable of a generating a temperature gradient across the plate.

2. A UV transilluminator or fluorescence gel scanner if fluorescence detection is used (optional).

3. Centrifuge with plate holders.

4. PCR plate or tubes with sealant or lids.

5. Suitable sample buffer, at the desired $\mathrm{pH}$ and ionic strength, such as PBSZ (as described above).

6. $5 \mathrm{X}$ native loading buffer: $5 \mathrm{ml}$ glycerol, $5 \mathrm{ml}$ ultrapure water, $10 \mathrm{mg}$ bromophenol blue.

7. $3-12 \%$ bis-Tris NativePAGE ${ }^{\text {TM }}$ gels with running buffer, or an equivalent native polyacrylamide gel system using Tris/Glycine buffers.

8. Coomassie or SYPRO Orange ${ }^{\mathrm{TM}}$ gel stain to visualise bands.

9. Plasma-derived or recombinant A1AT, unlabelled or labelled at the single cysteine with a fluorescein derivative (see Note 7).

\section{Polyacrylamide-agarose hybrid 'slab' gels}

1. Horizontal slab-gel DNA electrophoresis system, including tank, combs and gel casting box.

2. Fluorescence gel scanner.

3. $30 \%$ (37.5:1) acrylamide/bis-acrylamide solution (see Note 8).

4. $10 \mathrm{X}$ cathode buffer: $64 \mathrm{~g} \mathrm{~L}^{-1}$ Tris base, $51 \mathrm{~g} \mathrm{~L}^{-1}$ glycine in high purity water.

5. Tetramethylethylenediamine (TEMED).

6. Agarose powder suitable for DNA electrophoresis.

7. $10 \%$ ammonium persulfate solution, freshly prepared.

8. $5 \mathrm{X}$ native loading buffer: $5 \mathrm{ml}$ glycerol, $5 \mathrm{ml}$ ultrapure water, $10 \mathrm{mg}$ bromophenol blue.

9. SYPRO Orange ${ }^{\mathrm{TM}}$ gel stain.

10. $7.5 \%$ glacial acetic acid $+10 \%$ SDS in ultrapure water.

11. $7.5 \%$ glacial acetic acid in ultrapure water. 


\section{Methods}

\section{Preparation of fluorescent A1AT}

Sequestration of individual molecules into a growing polymer chain brings them into close proximity; therefore Förster resonance energy transfer (FRET) can be used to monitor the progress of the reaction in real-time. This requires two preparations of A1AT with different fluorescence properties to be prepared, using 'donor' and 'acceptor' fluorophore conjugates. Follow the protocol detailed here for each fluorophore (see Note 1).

1. Recombinant human A1AT (typically expressed in E. coli), or purified human plasma A1AT, harbours an endogenous cysteine at position 232. For optimal thiol conjugation efficiency, reduce the protein prior to introduction of a fluorescent conjugate (see Note 2) for 1030 minutes at room temperature with $10 \mathrm{mM}$ dithiothreitol.

2. Remove excess reducing agent using a subsequent $\geq 1000$-fold buffer exchange by dialysis. A maleimide conjugation reaction is most efficient around neutral $\mathrm{pH}$, thus phosphate buffered saline (PBSZ) is a suitable buffer to exchange the sample into. As an alternative to dialysis, anion exchange chromatography as described below can be performed (see also Note 3). If the sample is purified in this way, dilute $1: 3 \mathrm{v} / \mathrm{v}$ into PBSZ.

3. The concentration of a pure A1AT preparation can be determined by measuring the absorbance of a sample at $280 \mathrm{~nm}$ in a spectrophotometer, either cuvette-based or a device such as a NanoDrop ${ }^{\mathrm{TM}}$. The absorbance of a $1 \mathrm{mg} \mathrm{ml}^{-1}$ solution of plasmaderived A1AT in a $1 \mathrm{~cm}$ pathlength is 0.52 ; that of recombinant protein is 0.58 . Adjust the concentration to around $0.5-2 \mathrm{mg} \mathrm{ml}^{-1}$ in PBSZ by dilution or concentration using centrifugal concentrator units with a 10 or $30 \mathrm{kDa}$ cut-off.

4. Divide the protein to be labelled into two tubes. Protecting the reaction from light (see Note 9), add a 20 -fold molar excess of each fluorescent conjugate over the protein concentration. Incubate the samples at $25^{\circ} \mathrm{C}$ for 18 hours, in the dark. The incubation can be continued for an additional 24 hours with no adverse effect.

5. Quench the labelling reaction by addition of sufficient L-cysteine from a $1 \mathrm{M}$ stock solution to yield a concentration of $10 \mathrm{mM}$. Use a $0.45 \mu \mathrm{m}$ filter - either syringe-based or a centrifugal unit - to remove any precipitate.

6. Exchange the sample $\geq 1: 200$ into PBSZ using a centrifugal concentrator unit (preferably with a $30 \mathrm{kDa}$ cut-off). If available, anion exchange chromatography, described below, provides an effective means to remove most remaining unconjugated fluorophore.

7. The presence of unconjugated label can be determined by resolving the resulting samples using SDS-PAGE, and visualising with a UV transilluminator or fluorescence gel scanner (such as a Typhoon Trio, GE Healthcare) without staining. The free fluorophore will migrate close to the dye front. Determine the fluorescence intensity 
of the band near the dye front as a proportion of that of the labelled protein, using densitometry software such as ImageJ.

8. To minimise background in FRET experiments, unconjugated label would ideally be barely discernible but should comprise less than $10 \%$ of the protein-bound label. If the value is higher than this, perform another 1:200 buffer exchange into PBSZ using a new centrifugal concentrator unit.

9. Determine the conjugation efficiency from the molar ratio of conjugated label to protein, calculated from the absorbance of the sample at the fluorophore $\lambda_{\max }$ and at $280 \mathrm{~nm}$. These measurements are made using a spectrophotometer.

$$
\text { Efficiency }=\frac{\operatorname{Abs}_{\lambda \text { max }}}{\operatorname{Ext}_{\text {label }}} \times \frac{\operatorname{Ext}_{\mathrm{A} 1 \mathrm{AT}}}{\mathrm{Abs}_{280 \mathrm{~nm}}-\mathrm{Abs}_{\lambda \max } * p}
$$

where Ext $t_{\text {label }}$ is the extinction coefficient of the label at $\lambda_{\max }, p$ is the ratio of the absorbance of the label at $280 \mathrm{~nm}$ to its absorbance at $\lambda_{\max }$ (both parameters should be provided by the manufacturer), and Ext $_{\mathrm{A} 1 \mathrm{AT}}$ is $1.0 \times 10^{5} \mathrm{~cm}^{-1} \mathrm{M}^{-1}$ for plasma-derived A1AT and $7.8 \times 10^{4} \mathrm{~cm}^{-1} \mathrm{M}^{-1}$ for recombinant A1AT.

10. A poor labelling efficiency will disproportionately impact the intensity of the FRET signal. Therefore, for the experiments considered here, the labelling process should be repeated with freshly reduced material if the calculated value is below 0.7 .

11. Concentrate the labelled protein to around $1-2 \mathrm{mg} \mathrm{ml}^{-1}$, divide into aliquots, and freeze at $-80^{\circ} \mathrm{C}$ until use.

\section{Sample preparation using anion exchange chromatography}

This affinity chromatography step is useful to separate the A1AT preparation from contaminants such as reducing agent or unconjugated fluorophore. Size exclusion chromatography can also be used but has not been found to be as effective in the latter instance.

1. Connect a $1 \mathrm{ml}$ or $5 \mathrm{ml} \mathrm{Q}$ sepharose column to a chromatography system, 'purge' the tubing and pump with ultrapure water, and pass 3 column volumes of water through the column at the manufacturer's recommended flow rate.

2. Place the inlets of the chromatography system into anion exchange buffers $A$ and $B$, and 'purge' the system to ensure all tubing contains the appropriate buffer. Wash the column with 3 column volumes of buffer B (or until any residual material from previous experiments is eluted, as monitored by the absorbance at $280 \mathrm{~nm}$ ), and then equilibrate into buffer $A$.

3. Dilute the A1AT preparation into anion exchange buffer A to ensure an appropriate $\mathrm{pH}$ and low salt concentration. For samples in PBSZ, a 1:3 dilution is sufficient.

4. Apply the diluted sample to the column.

5. Remove unbound sample and buffer components (such as reducing agent) using 10 column volumes of anion exchange buffer $A$.

6. Apply a salt gradient to the column, from $0-50 \%$ buffer B across 16 column volumes. Use the fraction collector of the chromatography system to collect half-column volume fraction sizes of eluted material. The profile of the absorbance at $280 \mathrm{~nm}$ can be used to identify fractions of interest (see Note 10). 
7. Wash the chromatography system and Q sepharose column with ultrapure water, followed by $20 \%$ ethanol, for storage.

\section{Heat-induced polymerisation monitored by FRET}

Typically, depending on the presence of other destabilising factors such as mutations or denaturant, heating A1AT to a temperature in the vicinity of $50^{\circ} \mathrm{C}-65^{\circ} \mathrm{C}$ results in the formation of polymers in a matter of minutes to hours. Where the polymers contain subunits with fluorophores whose fluorescence profiles partially overlap, FRET can be used to monitor this process. Real-time thermal cyclers are ideally suited to this application, due to accurate temperature control, small sample requirements, and the ability of some to generate temperature gradients across a plate.

1. Select a real-time PCR plate that is suitable for use with the chosen thermal cycler. Devise a plate layout appropriate to the experimental aims. For example, the experiment may explore the effect of different temperatures, A1AT concentrations, or additives on the rate of polymerisation. If a thermal cycler is used that is capable of producing a temperature gradient across the plate, sample composition might be kept constant across columns and vary down rows.

2. Based on the conjugation efficiencies of the two fluorescent A1AT preparations, mix the samples such that the labels are in an equal ratio.

3. Dilute the combined sample to the working concentration in the chosen buffer, and dispense $20 \mu \mathrm{l}$ aliquots into wells. In our experience a concentration between $50 \mu \mathrm{g} \mathrm{ml}^{-1}$ and $200 \mu \mathrm{g} \mathrm{ml}^{-1}$ is a good starting place.

4. Seal the plate with transparent film suitable for real-time thermal cyclers, and centrifuge at $500 \mathrm{~g}$ for 1 minute.

5. If necessary, calibrate the thermal cycler for the individual fluorophores according to the instrument protocol (see Note 11). Create a constant-temperature (non-temperature cycling) programme of a suitable length for the thermal cycler, ensuring fluorescence is read at appropriate intervals (ideally 30 seconds at minimum). A heated lid should be used, as sample evaporation will affect the results. Heating and cooling rates should be set at their maximum. The fluorescence both of the donor and acceptor should be recorded.

6. Perform the polymerisation experiment.

7. Calculate the relative FRET efficiency by dividing the fluorescence of the acceptor by that of the donor for each time point, using an appropriate numerical package. This ratiometric calculation can reduce the impact of anomalies such as small bubbles. The resulting progress curves should show a sigmoidal increase, followed by a gradual decline in fluorescence (Figure 1). The half-time of polymerisation - the time at which the increasing signal reaches the midpoint between the maximum and minimum of the curve - is a convenient way to represent the rate of the reaction. This can be readily determined by scaling the FRET data to fall between 0 and 1 , and reading the time at which the value reaches 0.5 . 


\section{End-point experiments of polymerisation induced at multiple temperatures}

The susceptibility of an A1AT variant to polymerisation can be readily and rapidly assessed by incubating the material over a temperature range for a fixed period of time and visualising the result using native PAGE (Figure 2). This can be useful to obtain a qualitative comparison between variants, overcome sample heterogeneity (when coupled with western blot analysis), or to scout for appropriate conditions to undertake preparative polymerisation.

1. Dilute A1AT, either plasma-derived or recombinant, to $200 \mu \mathrm{g} \mathrm{ml}^{-1}$ in a suitable buffer, for example PBSZ.

2. Dispense $20 \mu \mathrm{l}$ sample aliquots into wells of a PCR plate (or PCR tubes) spanning the width of a thermal cycler heating block.

3. Cap the tubes or seal the plate. If necessary, centrifuge briefly at $500 \mathrm{~g}$ to ensure samples are at the bottom of the wells.

4. Program the thermal cycler to incubate with a single fixed-time step, consisting of a temperature gradient across the plate, for example between $45^{\circ} \mathrm{C}-60^{\circ} \mathrm{C}$. Durations might be 2,4 or 18 hours. Conclude with an indefinite $12^{\circ} \mathrm{C}$ step.

5. Insert the tubes/plate into the thermal cycler, and perform the incubation, using a heated lid to prevent evaporation.

6. The thermal cycler will have a facility to report the actual temperatures across the sample block; take note of these, as the distribution of values will not be linear.

7. To each well or tube, add $5 \mu \mathrm{l}$ of native loading buffer. In addition, prepare a monomer control: $20 \mu \mathrm{l}$ of $200 \mu \mathrm{g} \mathrm{ml}^{-1}$ with $5 \mu \mathrm{l}$ of native loading buffer.

8. The samples will be resolved by clear native PAGE. Commerciallyavailable Tris/Glycine or bis-Tris-based polyacrylamide gel systems work well, but Tris-Borate-EDTA (TBE) polyacrylamide gels do not. If using a system such as NativePAGE ${ }^{\text {TM }}$ (Life Technologies), do not use the tank dye additive that is used for blue native PAGE applications. Set up the gel running apparatus as described in the associated protocol. Load the gel with the monomer control, and heated samples. If using a 15 -well gel, add $15 \mu \mathrm{l}$ of each rather than the full $25 \mu \mathrm{L}$.

9. Electrophoresis is performed at $20-25 \mathrm{~mA}$ constant current for a single gel.

10. The protein can be visualised using a Coomassie blue-based stain, SYPRO Orange, or using a UV transilluminator if the material has been pre-labelled with a fluorophore. As an alternative, a western blot can be performed using an anti-A1AT antibody.

\section{Polyacrylamide-agarose hybrid 'slab' gels}

Polyacrylamide gels can be cast in a horizontal slab format to resolve proteins with an apparatus typically used for DNA electrophoresis. A larger number of samples can be processed than typically possible with vertical mini-gel systems, with around 60 wells for a $15 \times 20 \mathrm{~cm}$ gel format (Figure 3). Separation of A1AT polymers is according to molecular weight and charge, and use of an acrylamide-agarose mix ensures a strong gel matrix. Amounts reported are for a $100 \mathrm{ml}$ gel volume. Work in a fume hood, and ensure all casting components are to hand as the gel mixture sets rapidly. 
1. Place the gel mould in the casting case.

2. In a $250 \mathrm{ml}$ conical flask, add $16.7 \mathrm{ml}$ of a $30 \%$ acrylamide/bisacrylamide $(37.5: 1)$ solution, $10 \mathrm{ml} 10 \mathrm{X}$ cathode buffer, $22 \mathrm{ml}$ ultrapure water, and $80 \mu \mathrm{l}$ TEMED. Swirl gently.

3. In a separate conical flask prepare $50 \mathrm{ml}$ of a $2 \%$ agarose solution in ultrapure water, and boil in a microwave until dissolved. The solution should be monitored whilst heating to avoid boiling-over.

4. Allow the agarose to cool slightly before pouring the acrylamide solution into the agarose in a smooth motion, gently swirling until mixed.

5. Add $1 \mathrm{ml} 10 \%$ ammonium persulfate, swirling gently, and quickly transfer to the gel mould. Insert the combs to create the desired number of wells.

6. Allow the gel to set and cool, before placing in the electrophoresis tank. Fill the tank with $1 \mathrm{X}$ cathode running buffer so that the gel is submerged. For best results, run the gel at $4{ }^{\circ} \mathrm{C}$.

7. Add loading buffer to the protein samples, and carefully pipette them into the wells.

8. Run the gel at a constant $100 \mathrm{~V}-150 \mathrm{~V}$ for $2-2.5$ hours.

9. Gels can be stained using Coomassie blue for 2-4 hours followed by de-staining. Alternatively, SYPRO Orange $\mathrm{e}^{\mathrm{TM}}$ is a more effective stain for this gel format, used as detailed in steps 10-14.

10. Incubate gel in $7.5 \%$ acetic acid $+0.1 \%$ SDS for 2 hours at room temperature.

11. Rinse twice with $7.5 \%$ acetic acid to remove SDS.

12. Place gel in a solution of $7.5 \%$ acetic acid containing 1:5000 SYPRO Orange $^{\mathrm{TM}}$ overnight at room temperature, in the dark.

13. Rinse with $7.5 \%$ acetic acid for 30 minutes.

14. Record using a fluorescence scanner with an excitation around $490 \mathrm{~nm}$ and emission reading in the $590 \mathrm{~nm}-610 \mathrm{~nm}$ range.

\section{Notes}

1. Real-time thermal cycler instruments usually excite around $470 \mathrm{~nm}$, so a fluorescein-like 'donor' is used, such as Alexa Fluor ${ }^{\mathrm{TM}}$-488 (Life Technologies) or Atto ${ }^{\mathrm{TM}}$-488 (AttoTec). The 'acceptor' should have an overlap in excitation spectrum with the emission spectrum

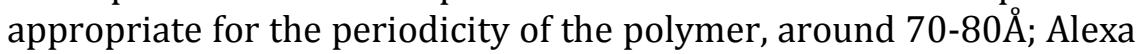
Fluor $^{\mathrm{TM}}$-594 (Life Technologies) and Atto ${ }^{\mathrm{TM}}$-594 (AttoTec) work well in this regard.

2. Plasma-derived A1AT can be obtained as a pharmaceutical preparation, as a research reagent, or can be conveniently purified using commercially available affinity resin (Antitrypsin Select, GE Biosciences).

3. During purification of recombinant or wild-type A1AT, it can be convenient to reduce the endogenous cysteine at position 232, in preparation for later conjugation. Typically the final step makes use of a $Q$ sepharose resin or gel filtration column. If, immediately prior to application on these columns, $10 \mathrm{mM}$ dithiothreitol is added to the sample, the purification will effectively remove the excess reducing agent. The sample should be frozen soon after elution.

4. If a chromatography system is not available, a 'gravity feed' method can be used in which loose resin at a final volume of $1-5 \mathrm{ml}$ is 
deposited into a column with a sintered glass frit, whose volume is several times that of the resin.

5. Buffers can be easily prepared from stock solutions of $1 \mathrm{M}$ Tris $\mathrm{pH}$ 8.0, $5 \mathrm{M} \mathrm{NaCl}$ and $20 \%$ sodium azide which have been filtered. If the ultrapure water is delivered through an inline filter (typical in modern systems), buffers can be prepared by mixing these components without filtering.

6. Some real-time thermal cyclers use detectors/filters that individually span very wide wavelength ranges. It is important that the fluorescence emission of the donor and that of the acceptor do not fall into the same detection 'bin'.

7. Fluorescein derivatives generally have an excitation spectrum that extends into the near UV region, and thus can be readily visualised using a UV transilluminator. The low background and linear response makes this a useful approach where densitometry is used.

8. Acrylamide is a neurotoxin with a cumulative effect; care must be exercised when handling and disposing of this chemical.

9. Fluorophores can be subject to photobleaching, although modern variants tend to be relatively photostable; provided they are not left unnecessarily exposed to light, we have not found it necessary to aliquot or mix in a darkened room. However, reactions and storage should be undertaken in the dark, for example by covering tubes with foil, and chromatography resins and eluted fractions should be shaded from light.

10. As fluorophores can often absorb at $280 \mathrm{~nm}$, resolve fractions by SDS-PAGE and visualise the gel with a UV transilluminator or fluorescence gel scanner (without staining). Unconjugated fluorophores will tend to run near the dye front.

11. If it is possible to export raw (non-deconvoluted) fluorescence data, this is generally preferred.

\section{Acknowledgements}

A.E.B. is supported by a Rosetrees Trust PhD studentship grant. I.H. is an eALTA Fellow. This work was funded in part by a grant from the Alpha-1 Foundation to J.A.I.

\section{Figure captions}

Figure 1. The polymerisation of A1AT as reflected by an increase in FRET. The graph shows the progress curves obtained during the polymerisation of fluorescently-labelled A1AT in a real-time thermal cycler. The curves were normalised by reporting fluorescence as a fold-increase over the starting FRET value. A temperature gradient was generated across the plate; the rate of polymerisation increases with increasing temperature, indicated by the arrow.

Figure 2. Polymerisation end-point experiment. Plasma-purified M A1AT was incubated for 18 hours over a $45.0^{\circ} \mathrm{C}-60.7^{\circ} \mathrm{C}$ temperature range, in PBS containing $5 \%$ glycerol. The resulting polymers were resolved by native PAGE and the protein visualised with Coomassie blue stain. The position of monomer is indicated by a triangle. 
Figure 3. Slab gel electrophoresis of polymers. The top half of an acrylamide-agarose slab gel, illustrating the ability to process a large number of samples at once. Different recombinant A1AT mutants were subjected to heat-induced polymerisation at $50^{\circ} \mathrm{C}$ for 18 hours, and resolved in duplicate according to the method described here. The leftmost lane shows monomeric recombinant A1AT, and the two right-most lanes wild-type polymer (labelled 'Mon').

Berg, N.O. and Eriksson, S. 1972. Liver disease in adults with alpha-1 -antitrypsin deficiency. N Engl J Med 287:1264-1267.

Bruch, M., Weiss, V., and Engel, J. 1988. Plasma serine proteinase inhibitors (serpins) exhibit major conformational changes and a large increase in conformational stability upon cleavage of their reactive sites. J Biol Chem 263:16626-16630.

Carrell, R.W. and Owen, M.C. 1985. Plakalbumin, a1-antitrypsin, antithrombin and the mechanism of inflammatory thrombosis. Nature 317:730-732.

Dafforn, T.R., Mahadeva, R., Elliott, P.R., Sivasothy, P., and Lomas, D.A. 1999. A kinetic mechanism for the polymerization of alpha1-antitrypsin. J Biol Chem 274:95489555.

Devlin, G.L., Chow, M.K., Howlett, G.J., and Bottomley, S.P. 2002. Acid Denaturation of alpha1-antitrypsin: characterization of a novel mechanism of serpin polymerization. Journal of molecular biology 324:859-870.

Ekeowa, U.I., Freeke, J., Miranda, E., Gooptu, B., Bush, M.F., Perez, J., Teckman, J., Robinson, C.V., and Lomas, D.A. 2010. Defining the mechanism of polymerization in the serpinopathies. Proc Natl Acad Sci USA 107:17146-17151.

Gooptu, B. and Lomas, D.A. 2009. Conformational pathology of the serpins - themes, variations and therapeutic strategies. Annu Rev Biochem 78:147-176.

Haq, I., Irving, J.A., Faull, S.V., Dickens, J.A., Ordonez, A., Belorgey, D., Gooptu, B., and Lomas, D.A. 2013. Reactive centre loop mutants of alpha-1-antitrypsin reveal position-specific effects on intermediate formation along the polymerization pathway. Biosci Rep 33.

Hidvegi, T., Schmidt, B.Z., Hale, P., and Perlmutter, D.H. 2005. Accumulation of mutant alpha1-antitrypsin $\mathrm{Z}$ in the endoplasmic reticulum activates caspases- 4 and -12 , NFkappaB, and BAP31 but not the unfolded protein response. J Biol Chem 280:39002-39015.

Irving, J.A., Miranda, E., Haq, I., Perez, J., Kotov, V.R., Faull, S.V., Motamedi-Shad, N., and Lomas, D.A. 2015. An antibody raised against a pathogenic serpin variant 
induces mutant-like behaviour in the wild-type protein. The Biochemical journal 468:99-108.

Kröger, H., Miranda, E., MacLeod, I., Pérez, J., Crowther, D.C., Marciniak, S.J., and Lomas, D.A. 2009. Endoplasmic reticulum-associated degradation (ERAD) and autophagy cooperate to degrade polymerogenic mutant serpins. J Biol Chem 284:22793-22802.

Lomas, D.A., Evans, D.L., Finch, J.T., and Carrell, R.W. 1992. The mechanism of Z alpha 1antitrypsin accumulation in the liver. Nature 357:605-607.

Lomas, D.A., Finch, J.T., Seyama, K., Nukiwa, T., and Carrell, R.W. 1993. Alpha 1antitrypsin Siiyama (Ser53-->Phe). Further evidence for intracellular loop-sheet polymerization. J Biol Chem 268:15333-15335.

Mallya, M., Phillips, R.L., Saldanha, S.A., Gooptu, B., Brown, S.C., Termine, D.J., Shirvani, A.M., Wu, Y., Sifers, R.N., Abagyan, R., and Lomas, D.A. 2007. Small molecules block the polymerization of $\mathrm{Z}$ alpha1-antitrypsin and increase the clearance of intracellular aggregates. Journal of medicinal chemistry 50:5357-5363.

Mast, A.E., Enghild, J.J., and Salvesen, G. 1992. Conformation of the reactive site loop of alpha 1-proteinase inhibitor probed by limited proteolysis. Biochemistry 31:2720-2728.

Miranda, E., Perez, J., Ekeowa, U.I., Hadzic, N., Kalsheker, N., Gooptu, B., Portmann, B., Belorgey, D., Hill, M., Chambers, S., Teckman, J., Alexander, G.J., Marciniak, S.J., and Lomas, D.A. 2010. A novel monoclonal antibody to characterize pathogenic polymers in liver disease associated with alpha1-antitrypsin deficiency. Hepatology 52:1078-1088.

Ordóñez, A., Pérez, J., Tan, L., Dickens, J.A., Motamedi-Shad, N., Irving, J.A., Haq, I., Ekeowa, U., Marciniak, S.J., Miranda, E., and Lomas, D.A. 2015. A single-chain variable fragment intrabody prevents intracellular polymerization of $\mathrm{Z} \alpha 1$ antitrypsin while allowing its antiproteinase activity. The FASEB Journal fj.14267351.

Ordóñez, A., Snapp, E.L., Tan, L., Miranda, E., Marciniak, S.J., and Lomas, D.A. 2013. Endoplasmic reticulum polymers impair luminal protein mobility and sensitize to cellular stress in alpha1-antitrypsin deficiency. Hepatology 57:2049-2060.

Powell, L.M. and Pain, R.H. 1992. Effects of glycosylation on the folding and stability of human, recombinant and cleaved a1-antitrypsin. J Mol Biol 224:241-252. 
Tsutsui, Y., Dela Cruz, R., and Wintrode, P.L. 2012. Folding mechanism of the metastable serpin alpha1-antitrypsin. Proceedings of the National Academy of Sciences of the United States of America 109:4467-4472.

Tsutsui, Y., Kuri, B., Sengupta, T., and Wintrode, P.L. 2008. The structural basis of serpin polymerization studied by hydrogen/deuterium exchange and mass spectrometry. J Biol Chem 283:30804-30811.

Yamasaki, M., Li, W., Johnson, D.J., and Huntington, J.A. 2008. Crystal structure of a stable dimer reveals the molecular basis of serpin polymerization. Nature 455:12551258.

Yamasaki, M., Sendall, T.J., Pearce, M.C., Whisstock, J.C., and Huntington, J.A. 2011. Molecular basis of alpha1-antitrypsin deficiency revealed by the structure of a domain-swapped trimer. EMBO reports 12:1011-1017. 


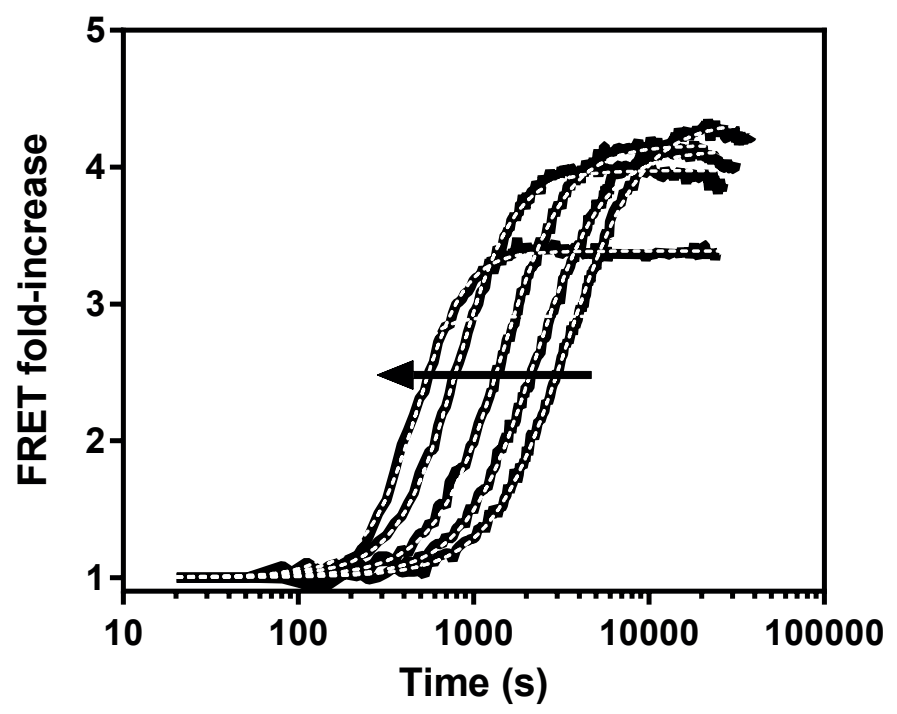

Figure 1

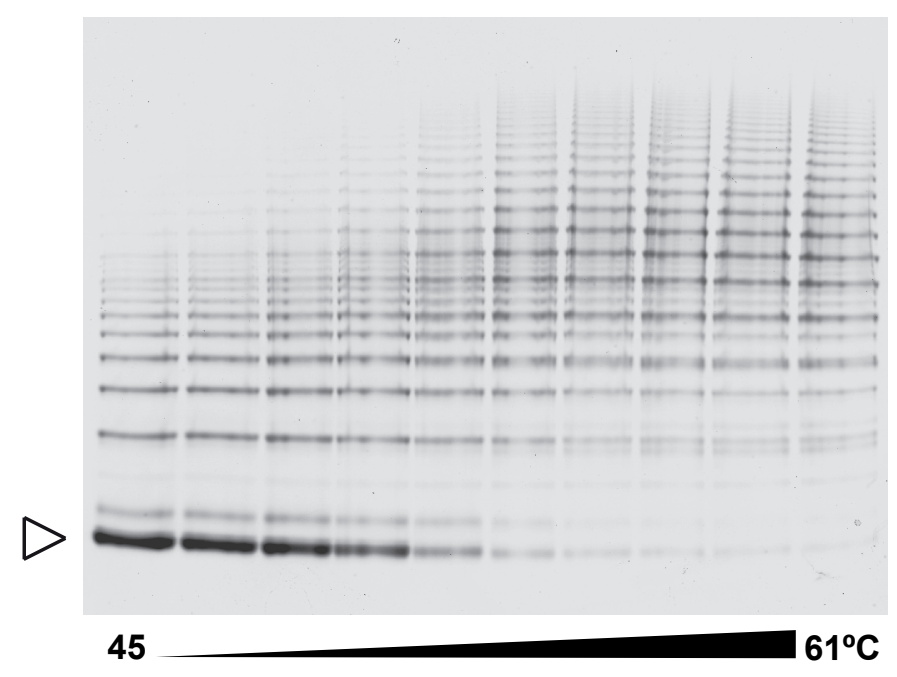

Figure 2

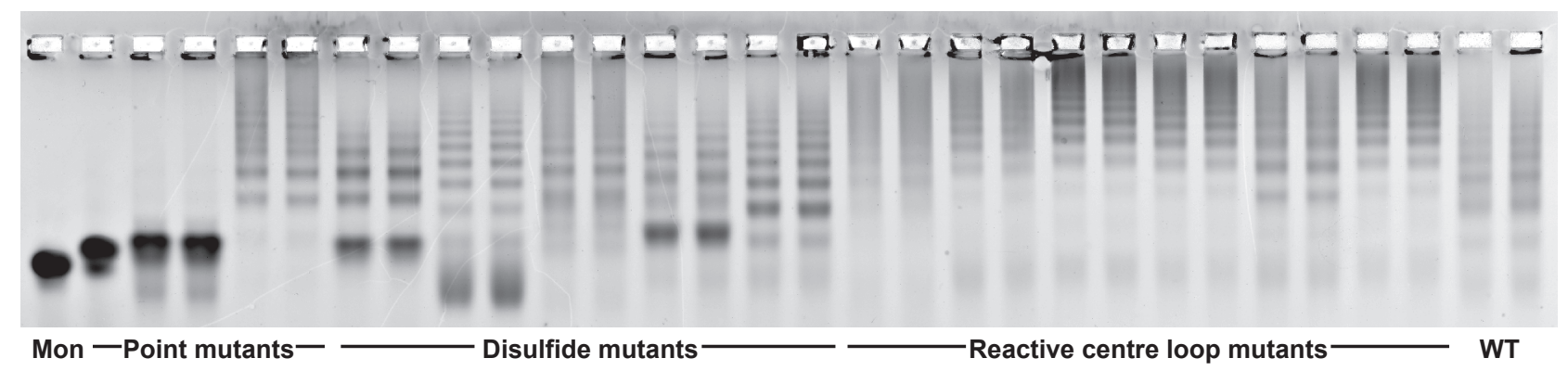

Figure 3 